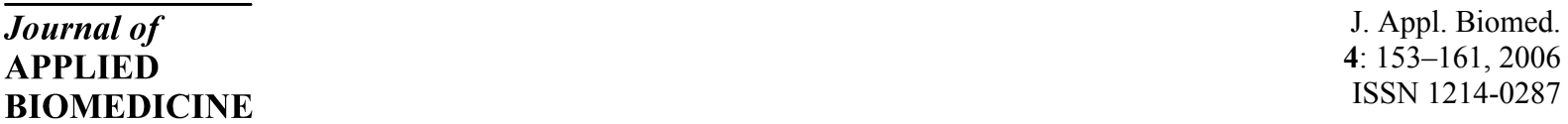

ORIGINAL ARTICLE

\title{
Enantiospecific pharmacokinetic studies on ketoprofen in tablet formulation using indirect chiral HPLC analysis
}

\author{
Kannappan Valliappan, Kamarajan Kannan, Thanikachalam Sivakumar, Rajappan Manavalan \\ Department of Pharmacy, Faculty of Engineering and Technology, Annamalai University, Annamalainagar, TN \\ 608002 , India
}

Received $21^{\text {st }}$ February 2006.

Revised $23^{\text {rd }}$ March 2006.

Published online $2^{\text {nd }}$ June 2006.

\begin{abstract}
Summary
The present study was undertaken to evaluate the possibility of chiral discrimination in the release of enantiomers of ketoprofen (KT) in tablet form and, in turn, the bioavailability of the individual enantiomers, using the rabbit as a model. The enantiomeric concentration of KT in plasma was determined using a customized chiral HPLC analysis. First the plasma concentration-time profile was established for pure $( \pm)$ KT in order to assess the extent of chiral discrimination. Subsequently the tablet formulation (Rhofenid-100mg) was administered, at a dose equivalent to $10 \mathrm{mg} / \mathrm{kg}$, to assess the enantiospecific bioavailability of KT enantiomers in the rabbit following the same protocol as followed for the pure KT. In vivo studies revealed that the bioavailability of S-KT is higher than that of the Renantiomer, after oral dosing with both KT-tablet and KT-pure. But the degree of chiral discrimination is more pronounced and more statistically significant in the case KT formulation as reflected by the enantioselective pharmacokinetic data. The observations presented in this article further emphasize the significance of differentiating between enantiomers of chiral drugs when assessing bioavailability and correlating efficiency with drug concentration. The study opens up a new avenue to the design of stereoselective dosage forms.
\end{abstract}

Keywords: ketoprofen enantiomers - enantiospecific - pharmacokinetic - bioavailability - chiral HPLC

Kannappan Valliappan, Department of Pharmacy, Faculty of Engineering and Technology, Annamalai University, Annamalainagar, TN 608 002, India

$\begin{array}{ll}\text { 虽 } & \text { kvalliappan@gmail.com } \\ \text { 且 } & +914144239738 \\ & +914144238145\end{array}$

\section{INTRODUCTION}

Over the last 15 years a great deal of attention has been paid to the effect of stereochemistry on drug action, metabolism and disposition (Jamali et al.1989, Caldwell 1995, Valliappan 1998, Somogyi et al. 2004, Patočka and Dvořák 2004). Excellent reviews are available describing stereoselectivity in the pharmacological, pharmacodynamic and pharmacokinetic behavior of chiral non-steroidal anti-inflammatory drugs (NSAIDs) (Adams et al. 1976, Crom 1992, Evans 1992). Ketoprofen, [KT, ( \pm )-2-(3-benzoylphenyl) propionic acid, Fig.1], is a member of the 2-aryl-propanoic acid series of 
NSAID, which has potent inhibitory effects on prostaglandin synthesis. It is commonly used in the treatment of rheumatoid arthritis and osteoarthritis. It is generally recognized that the S-profens are the enantiomers that inhibit prostaglandin synthetase. The configuration as well as the conformation of this enantiomer, S-ketoprofen, is regarded as the eutomer (the biological active enantiomer) of the profens and the R-form as the distomer (the biological inactive enantiomer) (Hutt and Caldwell 1984, Mullangi et al. 2003).

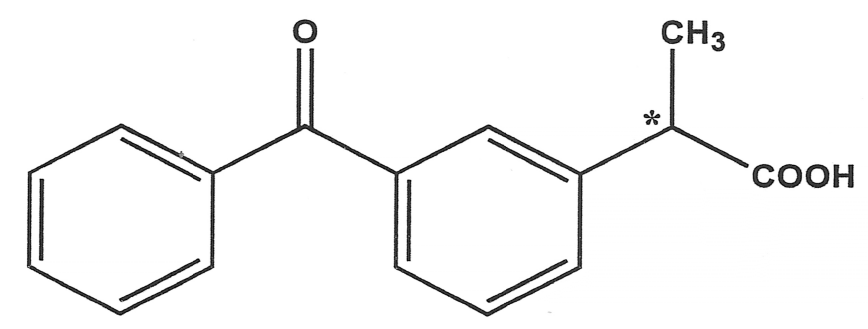

Fig. 1. Chemical structure of ketoprofen. The asterisk denotes the stereogenic center.

In practice, the profens are generally administered as a racemic mixture, except naproxen, which is administered in the $\mathrm{S}$-form because of the undesirable side effects of the Risomer and the burden on renal clearance (Strong 1999 and references therein). In vivo, however some of the profens can undergo, to a certain extent, a unidirectional inversion from $\mathrm{R}$ - to $\mathrm{S}$ form, leading to an enantiomeric excess of the Sform when a racemate of the drug is administered (Hutt and Caldwell 1983). Inversion has considerable therapeutic significance since antiinflammatory efficacy is mainly, if not exclusively, attributed to the S-enantiomers. Hence factors which affect the rate and extent of inversion are of prime importance. Studies have proved that there exists the possibility of chiral discrimination in the release of KT enantiomers from formulations providing a chiral environment (Duddu et al. 1993, Solinis et al. 2002, Valliappan et al. 2003) and thus resulting in enantioselective pharmacokinetics. Even though KT exhibits enantioselectivity in its action and disposition it is marketed as a racemate (Williams 1989). In this context, the enantiospecific investigation of the pharmacokinetic parameters of KT appears to be of scientific interest and relevant.

The objective of this study was to explore the possibility of chiral discrimination in the release of KT enantiomers from a chiral formulation: Rhofenid $100 \mathrm{mg}$ tablets of racemic KT. As only the S-enantiomer of KT possesses pharmacological activities it seems essential to examine the bioavailability of the active enantiomer. Thus a stereospecific HPLC assay was utilized to study the pharmacokinetics of the KT enantiomers in the rabbit after oral administration of racemic $\mathrm{KT}$ in pure and tablet (Rhofenid tablet powder) forms. The pharmacokinetic profiles of the release of KT enantiomers from the pure form and chiral formulation respectively were compared statistically to assess the degree and significance of enantiomeric discrimination.

\section{MATERIALS AND METHODS}

Chemicals and reagents

$( \pm)$-Ketoprofen and commercially available Rhofenid tablets containing ketoprofen-100 mg were gifted from Rhone-Poulenc, Mumbai, India. Probenecid (internal standard) and L-Leucinamide hydrochloride were purchased from Sigma (India office, Bangalore); Ethyl chloroformate was procured from Fluka (Buchs, Switzerland). Acetonitrile and methanol were HPLC grade while buffer salts and all other reagents employed were of analytical grade supplied by $\mathrm{M} / \mathrm{S} \mathrm{SD}$ Fine chemicals (Mumbai, India). HPLC-grade water was prepared using Milli-Q Academic, Millipore (Bangalore, India).

\section{Chromatographic apparatus and conditions}

The analysis was performed on a Shimadzu chromatographic system (Japan), equipped with an LC-10 ADvp solvent delivery Module, SPD-10 A UV-Visible detector, a Rheodyne model 7125injector valve fitted to a $20 \mu \mathrm{l}$ volume sample loop 
and a Shimadzu chromatographic work station CLASS LC 10 Ver. 1.63. The mobile phase consisted of acetonitrile and $10 \mathrm{mM}$ dipotassium hydrogen phosphate $(48: 52)$, with $\mathrm{pH}$ adjusted to 6.5 with phosphoric acid, pumped at a flow rate of $1.2 \mathrm{ml} / \mathrm{min}$. Diastereomers of KT were resolved on a Supelcosil ODS analytical column $(25 \times 0.46 \mathrm{~cm}$ I.D., $5 \mu \mathrm{m}$ particle size) in the reversed phase partition chromatographic condition. The system was used in an air-conditioned HPLC laboratory atmosphere $\left(20 \pm 2{ }^{\circ} \mathrm{C}\right)$. Before the analysis, the mobile phase was degassed using a Branson sonicator (Branson Ultrasonics Corporation, USA) and filtered through a $0.2 \mu$ filter (Gelman science, India). Sample solutions were also filtered through a $0.2 \mu$ filter. The system was equilibrated before injections were made. The column was monitored for UV absorbance at a wavelength of $256 \mathrm{~nm}$.

\section{Sample preparation}

The method of Foster and Jamali (Foster and Jamali 1987) was adopted with a minor modification for the extraction of KT from the plasma samples. Briefly, the method involves acidifying $200 \mu \mathrm{l}$ of plasma sample containing KT and $100 \mu \mathrm{l}$ of 15 $\mu \mathrm{g} / \mathrm{ml}$ Probenecid (I.S), with $100 \mu \mathrm{l}$ of $0.6 \mathrm{M}$ sulphuric acid. The resultant was extracted with $3 \mathrm{ml}$ of a mixture of isooctane: isopropanol (95:5) after vortex mixing for 30 seconds and centrifuging on a laboratory centrifuge $\left(\mathrm{Remi}^{\circledR}, \mathrm{R} \& \mathrm{C}\right.$, Remi Equipment, Mumbai, India) at $3500 \mathrm{rpm}(1878 \times \mathrm{g})$ for $5 \mathrm{~min}$. The aqueous phase was then discarded and the remaining organic phase $(2.5 \mathrm{ml})$ was evaporated to dryness by using the Eppendorf concentrator 5301 (Hamburg, Germany). The residue of $( \pm)$-KT was then derivatized according to the procedure described by Bjorkman (Bjorkman 1985, 1987). The residue obtained was reconstituted in $100 \mu \mathrm{l}$ of $50 \mathrm{mM}$ triethylamine in acetonitrile. To this mixture were added, at 30 seconds intervals, $50 \mu \mathrm{l}$ of $60 \mathrm{mM}$ ethyl chloroformate in acetonitrile and $50 \mu \mathrm{l}$ of $1 \mathrm{M} \mathrm{L}$ Leucinamide hydrochloride in methanol containing $1 \mathrm{M}$ triethylamine. After 2 minutes, $50 \mu \mathrm{l}$ of HPLC grade water were added. Aliquots of $20 \mu 1$ of these derivatized samples were chromatographed using reported optimized indirect chiral HPLC method (Valliappan 2002).

\section{Extraction recovery}

To assess the efficiency of the extraction method, spiked plasma solutions of racemic KT (5.0 and 10 $\mu \mathrm{g} / \mathrm{ml}$ ) were extracted according to the above procedure, but with the addition of IS after extraction. The extraction efficiency for the diastereoisomers was measured by comparison of the areas of derivatized drug injected directly into the reversed phase HPLC with those obtained after injection of the extracted samples. The recoveries were calculated as the area ratio of either R- or SKT to IS using the formula:

$$
\text { Recovery (\%) }=\frac{P_{\text {KTextr }} / I S}{P_{\text {KTnon-extr }} / I S} \times 100
$$

where $\mathrm{P}_{\mathrm{KT} \text { extr }} / \mathrm{IS}, \mathrm{P}_{\mathrm{KT} \text { non-extr }} / \mathrm{IS}$ are the peak area of extracted or non-extracted KT enantiomers to the peak area of IS, respectively.

\section{Enantiospecific pharmacokinetic studies}

Six healthy rabbits of either sex, weighing $1.9 \pm 0.2$ $\mathrm{kg}$, bred in the Central Animal House, Rajah Muthiah Medical College, Annamalai University, were used. The animals were housed in polycarbonate cages in a room with a $12 \mathrm{~h}$ daynight cycle, temperature of $25 \pm 1^{\circ} \mathrm{C}$ and humidity of 44-65\%. During the whole experimental period, the animals were fed with a standard pelleted diet (M/S Hindustan Lever Ltd., Mumbai, India) and water $a d$ libitum. All animal experiments were approved by the ethical committee, Annamalai University and were in accordance with the guidelines of the National Institute of Nutrition (NIN), Indian Council of Medical Research (ICMR), Hyderabad, India.

The study had an open, randomized, 2-period crossover design with a 7-day washout period between doses. Rabbits were fasted overnight $(\sim 14$ h) before the dosing day and they had free access to water throughout the experimental period. Initially during the $1^{\text {st }}$ period of the study, pure $( \pm)$-KT was administered orally at a dose of $10 \mathrm{mg} / \mathrm{kg}$ to assess the degree of inversion in the selected animal model. Blood samples were collected from the marginal ear vein at different time points $(0.0 .25$ $0.50,0.75,1.0,1.25,2.0,3.0,5.0,8.0$ and $12.0 \mathrm{~h}$ ) in tubes containing heparin sodium as anticoagulant. Plasma was harvested by centrifuging on a laboratory centrifuge at $3000 \mathrm{rpm}(1610 \times \mathrm{g})$ for 10 min and stored at $-20{ }^{\circ} \mathrm{C}$ until bioanalysis. The plasma samples $(200 \mu \mathrm{l})$ were spiked with I.S., extracted, re-dissolved, derivatized and injected as described before. During the $2^{\text {nd }}$ period of the study, $( \pm$ )-KT tablet powder (Rhofenid-100 mg) at a dose equivalent to $10 \mathrm{mg}( \pm)-\mathrm{KT}$ per $\mathrm{kg}$ was administered orally following the same protocol to assess the stereospecific bioavailability of $\mathrm{KT}$ enantiomers in the rabbit.

\section{Data analysis}

A standard calibration curve was constructed separately for the R- and S-KT enantiomers, using the $( \pm)-\mathrm{KT}$ covering the appropriate ranges and used to quantify both the enantiomers of KT in the rabbit plasma. The stock and working standard solutions of $( \pm)-\mathrm{KT}$ and Probenecid (I.S) were prepared in methanol: water $(80: 20)$, protected 
from light and stored at $-20{ }^{\circ} \mathrm{C}$ until used. Calibration standards were obtained by spiking drug free plasma with known amounts of $( \pm)$-KT in triplicate to achieve plasma concentrations of 0.05 , $0.1,0.25,0.5,1.0,2.5,5.0,10.0$ and $20.0 \mu \mathrm{g} / \mathrm{ml}$ of sseach enantiomer. The linearity was assessed for the peak area of R- and S-KT enantiomer/IS ratio as a function of each enantiomer concentration. The correlation coefficient $r^{2}$ was calculated to confirm the linearity of the calibration curves.

\section{Pharmacokinetic and statistical analysis}

The enantioselective kinetic disposition of KT was determined based on the standard noncompartmental model (Gibaldi 1991). The peak plasma concentration $\left(\mathrm{C}_{\max } ; \mathrm{mg} / \mathrm{l}\right)$ and time to reach peak plasma concentration $\left(\mathrm{t}_{\max } ; \mathrm{h}\right)$ was obtained from the visual inspection of the plasma concentration-time curves. The areas under the plasma concentration curve $\left(\mathrm{AUC}_{0-\mathrm{t}} ; \mathrm{mg} \mathrm{h} / \mathrm{l}\right)$ were determined using the trapezoidal rule up to $12 \mathrm{~h}$ post drug administration. The apparent terminal rate constant $\left(\mathrm{K}_{\mathrm{el}}\right)$ was calculated by the linear regression of the log-transformed concentrations of the drug in the terminal phase. The half-life $\left(t_{1 / 2}\right)$ of the terminal elimination phase was obtained using the relationship $\mathrm{t}_{1 / 2}=0.693 / \mathrm{K}_{\mathrm{el}}$.

The statistical significance of the observed differences between the concentration of enantiomers as well as between the pharmacokinetic indices was examined by paired $t$ test using SPSS version 9.5 (SPSS, Cary, NC, USA). Differences between means were considered statistically non-significant (NS) if the $P$ value was $>0.05$. When $0.05>P \geq 0.01$ the parameters were taken as significantly different $(\mathrm{S})$ and when $0.01>\mathrm{P} \geq 0.001$ they were regarded as highly significantly different (HS). The $S / R$ ratios ( $S / R$ at $\mathrm{T}_{\max }, \mathrm{S} / \mathrm{R}$ AUC ratio and $\mathrm{S} / \mathrm{R}$ concentration ratio) were determined from the results obtained and are taken as the measure for chiral discrimination. If this ratio is close to unity it can be hypothesized that there is no difference in the pharmacokinetic parameters and therefore no chiral discrimination between KT enantiomers.

Table 1. Bioavailability indices following oral administration of $10 \mathrm{mg} / \mathrm{kg}( \pm)-\mathrm{KT}$ in the form of pure and tablet powder (RHOFENID-100 mg)

\begin{tabular}{|c|c|c|c|c|c|c|c|c|c|c|}
\hline \multirow{2}{*}{$\begin{array}{c}\text { Animal } \\
\text { No. }\end{array}$} & \multirow{2}{*}{$\mathrm{T}_{\max }^{\mathrm{a}}, \mathrm{h}$} & \multicolumn{2}{|c|}{$\mathrm{C}_{\max }, \mathrm{mg} / \mathrm{l}$} & \multirow{2}{*}{$\mathrm{S} / \mathrm{R}$ at $\mathrm{T}_{\max }$} & \multicolumn{4}{|c|}{ AUC, mg h/l } & \multicolumn{2}{|c|}{$\mathrm{t}_{1 / 2}, \mathrm{~h}$} \\
\hline & & S & $\mathrm{R}$ & & S & $\mathrm{R}$ & $\mathrm{S} / \mathrm{R}$ & $\mathrm{S} / \mathrm{R}$ conc. ratio & S & $\mathrm{R}$ \\
\hline \multicolumn{11}{|l|}{ KT pure } \\
\hline 1 & 0.75 & 18.46 & 4.75 & 3.89 & 54.87 & 10.43 & 5.26 & 60.16 & 2.70 & 3.39 \\
\hline 2 & 0.50 & 21.90 & 6.15 & 3.56 & 77.45 & 15.19 & 5.10 & 56.66 & 2.71 & 3.23 \\
\hline 3 & 0.50 & 17.58 & 5.12 & 3.43 & 50.48 & 10.50 & 4.81 & 57.42 & 2.27 & 2.61 \\
\hline 4 & 0.75 & 20.56 & 5.32 & 3.86 & 64.42 & 11.84 & 5.44 & 58.74 & 2.38 & 3.04 \\
\hline 5 & 1.00 & 16.35 & 3.98 & 4.11 & 60.67 & 11.04 & 5.50 & 62.35 & 2.87 & 3.57 \\
\hline 6 & 0.75 & 17.46 & 4.31 & 4.05 & 58.04 & 11.06 & 5.25 & 55.65 & 2.53 & 3.32 \\
\hline Mean & 0.71 & 18.72 & 4.94 & 3.82 & $60.99^{\dagger}$ & 11.68 & 5.23 & 58.50 & $2.58^{\circ}$ & 3.19 \\
\hline SD & 0.19 & 2.10 & 0.77 & 0.27 & 9.37 & 1.79 & 0.25 & 2.46 & 0.23 & 0.34 \\
\hline \multicolumn{11}{|l|}{ KT tablet } \\
\hline 1 & 1.00 & 19.56 & 4.35 & 4.50 & 59.48 & 6.90 & 8.62 & 124.08 & 2.63 & 2.47 \\
\hline 2 & 0.75 & 22.47 & 5.52 & 4.07 & 80.64 & 9.81 & 8.22 & 118.88 & 2.92 & 2.86 \\
\hline 3 & 0.75 & 17.60 & 4.17 & 4.22 & 52.72 & 6.44 & 8.19 & 118.56 & 2.43 & 2.28 \\
\hline 4 & 0.75 & 21.57 & 4.56 & 4.73 & 72.16 & 5.92 & 12.19 & 119.20 & 2.56 & 2.49 \\
\hline 5 & 1.00 & 17.86 & 3.02 & 5.91 & 69.89 & 8.27 & 8.45 & 107.86 & 3.04 & 3.12 \\
\hline 6 & 1.00 & 18.57 & 3.12 & 5.95 & 68.40 & 7.61 & 8.99 & 114.58 & 2.68 & 2.64 \\
\hline Mean & $0.88^{\mathrm{S}}$ & $19.61^{\mathrm{HS}}$ & $4.12^{\mathrm{HS}}$ & $4.90^{\mathrm{HS}}$ & $67.22^{\mathrm{HS} \dagger}$ & $7.49^{\mathrm{HS}}$ & $9.11^{\mathrm{HS}}$ & $117.19^{\mathrm{HS}}$ & $2.71^{\mathrm{S}}$ & $2.64^{\mathrm{HS}}$ \\
\hline SD & 0.14 & 2.01 & 0.94 & 0.83 & 9.83 & 1.41 & 1.54 & 5.48 & 0.23 & 0.30 \\
\hline
\end{tabular}

Differences between enantiomers of KT pure and KT Tablet: NS, non-significant $(\mathrm{P}>0.05)$; S, significant $(0.05>\mathrm{P} \geq 0.01)$; HS, highly significant $(0.01>\mathrm{P} \geq 0.001) ;{ }^{\dagger}$ Highly significant from $\mathrm{AUC}(\mathrm{R}) ;{ }^{\circ}$ Highly significant from $\mathrm{t}_{1 / 2}(\mathrm{R}) ;{ }^{\mathrm{a}}$ Time to attain $\mathrm{C}_{\max }$ of the $\mathrm{S}$ enantiomer. 


\section{RESULTS AND DISCUSSION}

In Fig. 2 are depicted the representative chromatograms of drug-free plasma, plasma spiked with $10 \mu \mathrm{g} / \mathrm{ml}( \pm)-\mathrm{KT}$, and rabbit plasma sample taken $30 \mathrm{~min}$ after administration of a $10 \mathrm{mg} / \mathrm{kg}$ oral dose of $( \pm)-\mathrm{KT}$ in the form of pure drug and Rhofenid tablet powder. Typical chromatograms of drug-free rabbit plasma and a plasma sample from a healthy rabbit collected $30 \mathrm{~min}$ after the oral administration of a $10 \mathrm{mg} / \mathrm{kg}( \pm)-\mathrm{KT}$ dose show that KT enantiomers were clearly separated without interference from endogenous compounds from the matrix. The extraction efficiencies of KT diastereoisomers were $61.4 \pm 5.9$ and $64.5 \pm 7.1$ for the S-enantiomer and $63.8 \pm 5.2$ and $66.8 \pm 6.1$ for the R-enantiomer at $( \pm)$-KT concentrations of 5 and 10 $\mu \mathrm{g} / \mathrm{ml}$, respectively. The retention times for the diastereoisomers of R- and S-KT were approximately 5.6 and $6.2 \mathrm{~min}$ respectively and while those of IS was $8.1 \mathrm{~min}$.

A typical plasma standard curve could be described by $\mathrm{y}=0.2 \mathrm{x}-0.0104$ and $\mathrm{y}=0.2048 \mathrm{x}-0.0228$ for the R- and S-KT enantiomers, respectively. The average regression $(n=3)$ was 0.9997 and 0.9989 for R- and S-KT respectively. Excellent linearity was observed between the peak area ratios (R- and S-KT:IS) (y) and the corresponding plasma concentrations $(x)$ in the examined concentration range of $0.05-20.0 \mu \mathrm{g} / \mathrm{ml}\left(\mathrm{r}^{2}>0.998\right)$. As reflected by the slope of the best-fit lines, the responses to both enantiomers were close, indicating nonstereoselectivity in the extraction and/or the derivatization methods. Equations of the standard curves manifested a relatively low value for the intercept, not significantly different from zero.

Fig. 3A depicts the plasma course of enantiomers after the administration of a single dose of $10 \mathrm{mg} / \mathrm{kg}$ of pure $( \pm)-\mathrm{KT}$. It can be observed that the levels of AUC (S) were significantly higher $(0.01>\mathrm{P} \geq 0.001)$ than those of AUC (R), which suggest that an inversion from Rto S-KT might occur. AUC (S) was 5.23-fold higher than that for AUC (R), which confirms the stereoselective disposition of KT (Table 1). Because of the fact that the differences in plasma concentration time profiles between R- and S-KT are already observed $15 \mathrm{~min}$ after an oral administration of $\mathrm{KT}$, it is likely that they are originated from the occurrence of a presystemic chiral inversion from R- to S-KT.

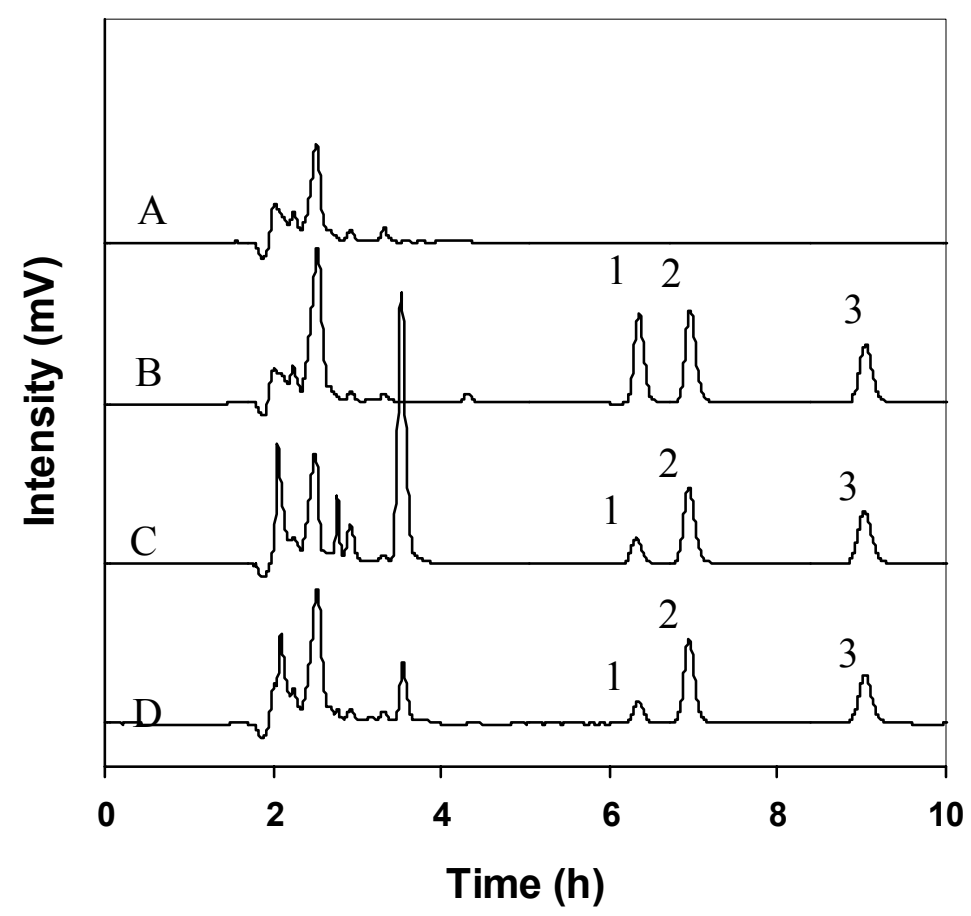

Fig. 2. Representative chromatograms of (A) blank rabbit plasma, (B) blank rabbit plasma spiked with 10 $\mu \mathrm{g} / \mathrm{ml}( \pm)-\mathrm{KT},(\mathrm{C})$ rabbit plasma sample collected $30 \mathrm{~min}$ after administration of a $10 \mathrm{mg} / \mathrm{kg}$ oral dose of pure $( \pm)$-KT and (D) rabbit plasma sample collected $30 \mathrm{~min}$ after administration of a $10 \mathrm{mg} / \mathrm{kg}$ oral dose of Rhofenid tablet powder. Peaks: 1, R- and 2, S-KT diastereoisomers; 3, Probenecid (I.S). 
A
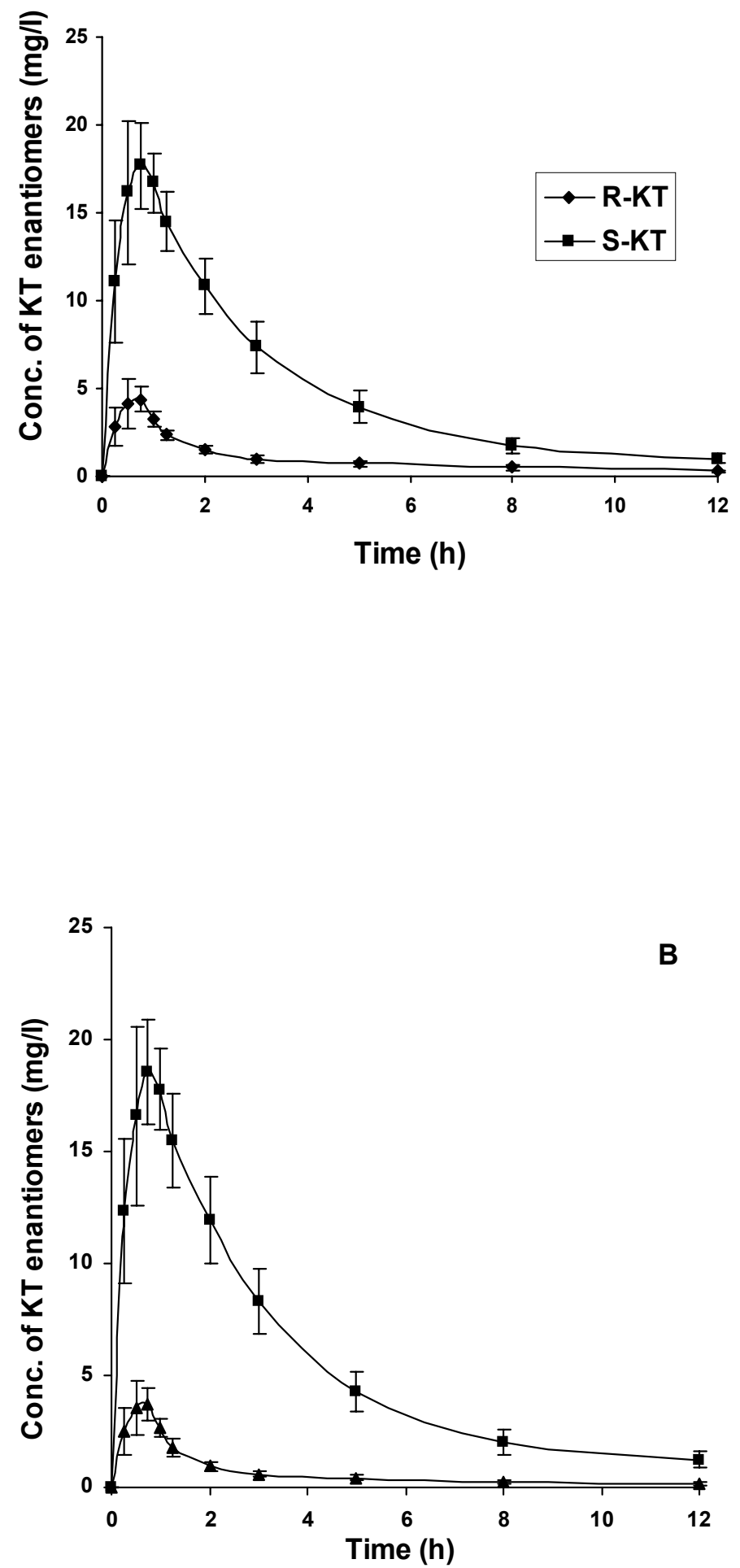

Fig. 3. Mean plasma concentration-time curves of KT enantiomers following oral administration of 10 $\mathrm{mg} / \mathrm{kg}$ of $(\mathrm{A}) ;( \pm)$-KT pure and $(\mathrm{B}) ;( \pm)$-KT tablet powder (Rhofenid-100 $\mathrm{mg}$ ) in rabbits. Each point represents the mean \pm S.D. of 6 values corresponding to 6 rabbits. 


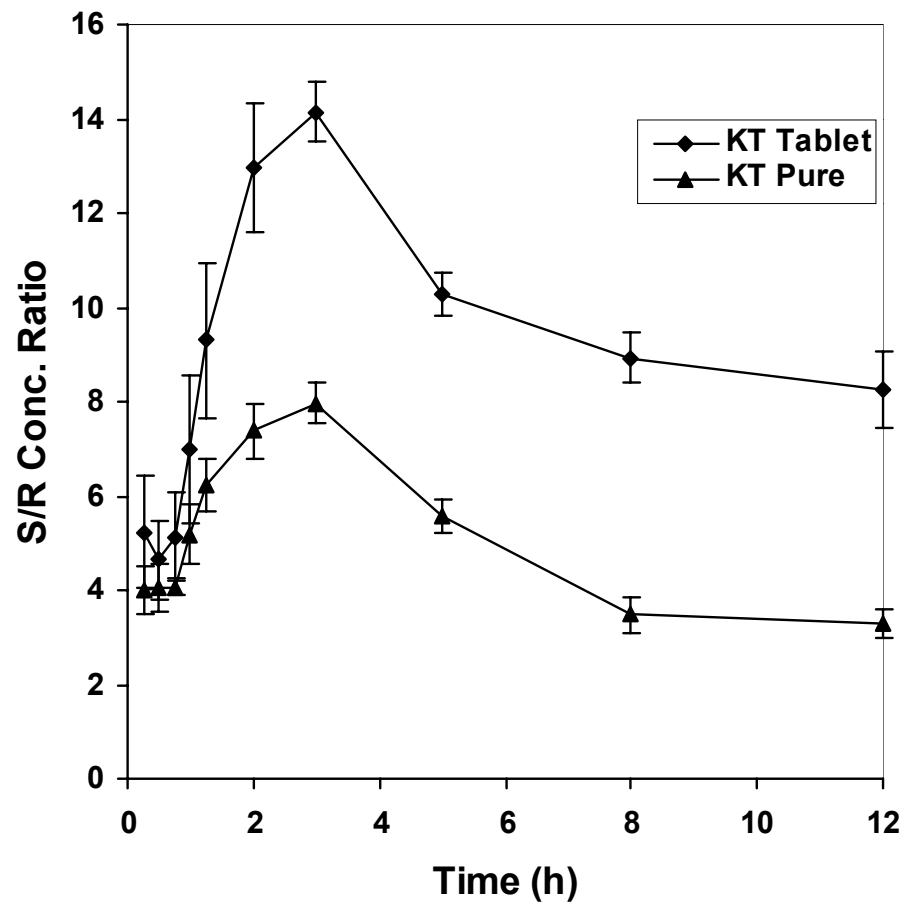

Fig. 4. S/R concentration ratios versus time curves following oral administration of $10 \mathrm{mg} / \mathrm{kg}( \pm)-\mathrm{KT}$ in the form of pure and tablet powder (Rhofenid-100 mg) in rabbits. Each point represents the mean \pm S.D. of 6 values corresponding to 6 rabbits.

It is noticed that the $\mathrm{S} / \mathrm{R}$ concentration ratio increased progressively until 2-4 h post dosing and then leveled off, which, although not conclusive, leads us to propose that R- to S-KT inversion takes place presystemically (Fig. 4). Since systemic inversion is supposed to be a continuous process, in its presence one should expect the concentration gap between the two isomers to broaden. But we noticed a parallel decline in the plasma concentrations of the enantiomers. Further, the differences between enantiomers could also be noted during the elimination phase. In the presence of systemic inversion from $\mathrm{R}$ to $\mathrm{S}$, one would expect higher values of $t_{1 / 2}$ for $\mathrm{S}$ - than for $\mathrm{R}$ enantiomer. But significantly higher values $(0.01>\mathrm{P} \geq 0.001)$ of $t_{1 / 2}$ were noted for $\mathrm{R}$ enantiomer than for S-enantiomer $\left[t_{1 / 2}\right.$ $(\mathrm{R})=3.19 \pm 0.34 \mathrm{~h}$ and $\left.t_{1 / 2}(\mathrm{~S})=2.58 \pm 0.23 \mathrm{~h}\right]$. The above observations exclude the possibility of systemic inversion. To confirm the above hypothesis, the administration of i.v. doses and more detailed experiments on the absorption from the gut and the inversion mechanism after administration of R-KT, S-KT and ( \pm )-KT must be undertaken. .
Fig. 3B depicts the plasma course of enantiomers after the administration of $10 \mathrm{mg} / \mathrm{kg}$ of KT-tablet powder. The AUC (S) were significantly greater $(0.01>\mathrm{P} \geq 0.001)$ than AUC (R) $(9.11$-fold) but the difference is well pronounced when compared to administration of the pure KT (Table 1). The $S / R$ concentration ratios at $T_{\max }$ and $A U C$ $(\mathrm{S} / \mathrm{R})$ concentration ratios (Table 1) were found to be significantly greater $(0.01>P \geq 0.001)$ after the administration of the KT-tablet compared to pure KT. Similarly, significant differences were found in $\mathrm{C}_{\max }$ values of either enantiomers between administration of the KT-tablet and pure KT.

Further, it is noticed that the $\mathrm{T}_{\max }$ of $\mathrm{S}$ enantiomer $(0.88 \pm 0.14)$, from the KT-tablet, is significantly high $(0.05>\mathrm{P} \geq 0.01)$ compared to that of the pure form $(0.71 \pm 0.19)$, suggesting that the rate of absorption of the S-enantiomer from KTtablet is relatively slow. This observation could probably be due to the following causes: (i) slow release of R-enantiomer from the chiral matrices of $\mathrm{KT}$-tablet (ii) a gradual bio-inversion process, $\mathrm{R}$ to $\mathrm{S}-\mathrm{KT}$, in GIT.

In the presence of presystemic inversion, the longer R-KT resides in the absorption site, the greater will be its extent of inversion to the active 
S-enantiomer. So, the concentration of the active Senantiomer is influenced by the rate of absorption: the slower the rate of absorption the greater the extent of inversion. This could explain the significantly greater $(0.01>\mathrm{P} \geq 0.001)$ AUC of the $\mathrm{S}$ enantiomer after the administration of the KT-tablet compared to pure KT. As expected, a corresponding reduction in AUC of the Renantiomer was also noted $(0.01>P \geq 0.001)$. The differences in enantiomer levels between the KTtablet and bulk KT could also be noted during the elimination phase. Significantly higher values $(0.01>\mathrm{P} \geq 0.001)$ of $\mathrm{t}_{1 / 2}$ were noted for $\mathrm{R}$-enantiomer following the administration of the pure $\mathrm{KT}\left(t_{1 / 2}\right.$ $(\mathrm{R})=3.19 \pm 0.34 \mathrm{~h}$ and $\left.t_{1 / 2}(\mathrm{~S})=2.58 \pm 0.23 \mathrm{~h}\right)$. On the other hand, slightly higher values of $t_{1 / 2}$ were noted for S-enantiomer following the KT-tablet $\left(\mathrm{t}_{1 / 2}\right.$ $(\mathrm{R})=2.64 \pm 0.30 \quad \mathrm{~h} \quad$ and $\left.\quad \mathrm{t}_{1 / 2} \quad(\mathrm{~S})=2.71 \pm 0.23 \mathrm{~h}\right)$, however, the differences proved statistically insignificant $(\mathrm{P}>0.05)$. The higher levels of $\mathrm{S}$ enantiomer following oral dosing with the KTtablet could be explained as a consequence of the combined effect of stereochemical interactions between KT-enantiomers and chiral excipient in KT-tablets and presystemic chiral inversion.

The study reveals that there is chiral discrimination in the bioavailability of $\mathrm{KT}$ enantiomers as reflected by a high enantiomeric ratio. The bioavailability data suggest that the enantiomeric discrimination is higher and more significant in the formulation as compared to the pure KT. The observed small but significantly greater AUC of the S enantiomer following the Rhofenid tablet as compared with pure $\mathrm{KT}$ is a valuable finding, since the anti-inflammatory activity resides only on the $\mathrm{S}$ enantiomer of KT. The study further emphasizes the need to carry out enantioselective investigations when bioavailability studies are done on racemic therapeutics.

In this case, the interaction of the chiral excipient with the ketoprofen racemate appears favorable by enhancing the bioavailability of the Senantiomer compared to R-enantiomer. Further by choosing the appropriate chiral excipient and formulation conditions one can deliberately manipulate the release of a specific enantiomer from a chiral drug. This deliberate stereospecific modification in the release of enantiomers could be exploited for the design of stereoselective solid dosage form.

\section{ACKNOWLEDGEMENTS}

This work is a part of the R \& D project funded by all India Council for Technical Education (AICTE), New Delhi, India (Project No.8020/RID/R\&D-99, 2001-2002). Authors would like to thank AICTE for the financial assistance.

\section{REFERENCES}

Adams S.S., Breshloff P., Mason C.G.: Pharmacological difference between the optimal isomers of Ibuprofen: evidence for metabolic inversion of the (-)-isomer. J. Pharm. Pharmacol. 28:256-257, 1976.

Bjorkman S.: Determination of the enantiomers of indoprofen in blood plasma by highperformance liquid chromatography after rapid derivatization by means of ethyl chloroformate. J. Chromatogr. 339:339-346, 1985.

Bjorkman S.: Determination of the enantiomers of ketoprofen in blood plasma by ion-pair extraction and high-performance liquid chromatography of leucinamide derivatives. J. Chromatogr. A. 414:465-471, 1987.

Caldwell J.: Chiral pharmacology and the regulation of new drugs. Chem. Ind. 6:176179, 1995.

Crom W.R.: Effect of chirality on pharmacokinetics and pharmacodynamics. Am. J. Hosp. Pharm. 59:S9-S14, 1992.

Duddu S.P., Vakilynejad M., Jamali F., Grant D.J.W.: Stereoselective dissolution of propranolol hydrochloride from hydroxypropyl methylcellulose matrices. Pharm. Res. 10:1648-1652, 1993.

Evans A.M.: Enantioselective pharmacodynamics and pharmacokinetics of chiral non-steroidal anti-inflammatory drugs. Eur. J. Clin. Pharmacol. 42:237-256, 1992.

Foster R.T., Jamali. F.: High-performance liquid chromatographic assay of ketoprofen enantiomers in human plasma and urine. J. Chromatogr. 426:388-393, 1987.

Gibaldi M.: Compartmental and noncompartmental pharmacokinetics. In Gibaldi M. (ed.): Biopharmaceutics and Clinical Pharmacokinetics, Lea and Febiger, Philadelphia, London 1991, pp.14-23.

Hutt A.J., Caldwell J.: The importance of stereochemistry in clinical pharmacokinetics of the 2-arylpropionic acid non-steroidal antinflammatory drugs. Clin. Pharmacokinet. 9:371-373, 1984.

Hutt A.J., Caldwell J.: The metabolic chiral inversion of 2-arylpropionic acids- a novel route with pharmacological consequence. Pharm. Pharmacol. 35:693-704, 1983.

Jamali F., Mehvar R., Pasutto F.M.: Enantioselective aspects of drug action and disposition: therapeutic pitfalls. J. Pharm. Sci. 78:695-715, 1989.

Mullangi R., Yao M., Srinivasan N.R.: Resolution of enantiomers of ketoprofen by HPLC: a review. Biomed. Chromatogr. 17:423-434, 2003.

Patočka J., Dvořák A.: Biomedical aspects of chiral molecules. J. Appl. Biomed. 2:95-100, 2004. 
Solinis M.A., Cruz Y.D.L., Calco B. et al.: Release of salbutamol and ketoprofen enantiomers containing HPMC and cellulose derivatives. Chirality 14:806-813, 2002.

Somogyi A., Bochner F., Foster D.: Inside the isomers: the tale of chiral switches. Aust. Prescr. 27:47-49, 2004.

Strong M.S.: FDA policy and regulation of stereoisomers: Paradigm shift and the future of safer, more effective drugs. Food Drug Law J. 54:463-487, 1999.

Valliappan K., Kannan K. Manavalan R., Muralidharan C.: Evaluation of stereoselective dissolution of racemic ketoprofen from formulations containing chiral excipients. Indian J. Pharm. Sci. 65:253-259, 2003.

Valliappan K., Kannan K., Manavalan R., Muralidharan C.: Prediction of chiral separation of ketoprofen using experimental design. Indian J. Chem. 41A:1334-1340, 2002.

Valliappan K.: Molecular handedness: Impact on the development of chiral drugs. Indian Drugs 35:446-457, 1998.

Williams K.M.: Clinical applications of enantiomeric drugs. Aust. Prescr. 12:22-25, 1989. 